\title{
Protozoa enumeration via microscope - some remarks on methodology
}

\author{
Sara Nicpoń $^{1}$, Paula Iliaszewicz $^{1^{*}}$, Maciej Leoniak ${ }^{1}$, and Agnieszka Trusz-Zdybek ${ }^{1}$ \\ ${ }^{1}$ Wroclaw University of Technology, Faculty of Environmental Engineering, Wybrzeże Stanisława \\ Wyspiańskiego 27, 50-370 Wrocław, Poland
}

\begin{abstract}
For proper enumeration of protozoa in activated sludge good methodology is required. In this paper we present some remarks on microscopic methodology of protozoa enumeration. This remarks concern number of repetitions from one sample required to obtain good statistical results as well as influence of sample aeration on number of found protozoa. Presented data shows that at last 10 repetitions are required from each sample to obtain low average confidence interval. Lower number of repetitions leads to sharp increase in average confidence interval and loss of statistical significance while higher number does not decrease average confidence interval substantially. As measurements lasts for few hours lack of sample's aeration during measurement leads to detection of lower by $27 \%$ number of protozoa.
\end{abstract}

\section{Introduction}

Protozoa are common component of activated sludge [1] and have influence on activated sludge [1]. Realiable methodology of protozoa enumaration is important for many fields of research such as nitrogen removal [2], clarification [1], process stability [3]. Most popular method is microscopic analysis of protozoa abundance.

\subsection{The problem and its importance}

In available literature at least a few methods of preforming protozoa enumeration exist with no detailed information about procedure (including aeration of main beaker) and statistic parameters. This makes comparison of reported values difficult. It is a common knowledge that in every sampling procedures the number of samples is very important and in general: the higher samples number is the better and more reliable results are obtained. Higher number of samples requires higher effort and it is not clear how many repetitions should be made for optimal results without unnecessary costs. Such information are not available at many publications $[2,4,5]$.

\footnotetext{
* Corresponding author: p.iliaszewicz@gmail.com
} 


\subsection{Aim of paper}

Main goal of paper is to show that some aspects of methodology of microscopic enumeration of protozoa have important influence on obtained results and procedures should be clear and unified in that aspects. Efficient research work requires founding compromise between workload and reliable results. It is nessescary to develop the smallest number of samples which will gives representative values. Moreover this minimal number of samples might be changed and depend by kind of experiment. Main aim of paper is develop optimal point for method of enumeration protozoa.

\section{Materials and methods}

\subsection{The general scheme of the experiment}

The experiment was carried out in two stages. The first step dealt with effect of aeration on the number of protozoa found in sample. The lack of aeration of beakers with sludge may result in the death of protozoa. In oxygen limiting conditions protozoa which are normally moving and are recognizable in the microscope lenses accumulate in the flocs and become invisible. As a result, the observations would be unreliable. For this test 10 samples were taken from aerated and non-aerated beaker.

The second stage was to examine the amount of samples needed for obtaining statistically reliable results. For that test, 3 times in different days sludge was examined with 16 samples taken each time. The general scheme of the experiment is shown in Fig. 1.

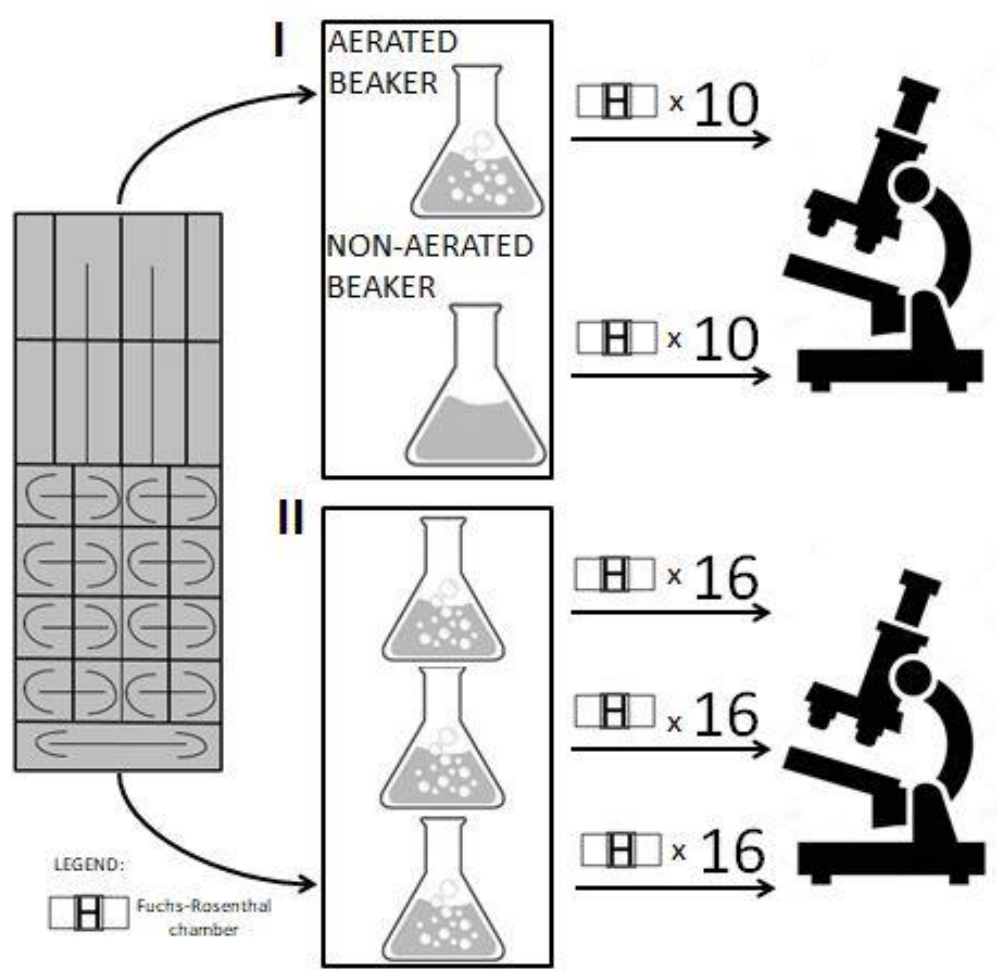

Fig. 1. Scheme of the experiment. 


\subsection{Counting methods for protozoa}

The material for the research was collected with a scoop from the activated sludge chambers. The material from chambers was placed in $250 \mathrm{~cm}^{3}$ beakers during measurements. The samples for microscopic examination were taken with a pipette and placed in a Fuchs-Rosenthal chamber. This chamber allows the counting of microorganisms in relation to the known, defined volume and therefore allows for enumeration of protozoa. The sample was covered with a coverslip. Microscopic observations were carried out under the Olympus CH30 microscope. During the observations, the overall number of protozoa was calculated.

\subsection{The influence of aeration in beakers during measurements}

The tests were carried out in two beakers with the same material. The first beaker was constantly aerated, while the second beaker was not. An aeration diffuser was placed in beaker. Temperature and oxygen concentration were controlled with the HACH LANGE LDO probe. Therefore, the conditions in the aerated beaker were still close to those prevailing on the biological block.

\subsection{Confidence interval of average protozoa number}

Determination of the actual population protozoa in the activated sludge requires analysis of the entire volume of the reactor. It is impossible. Taking into account availability of only a limited sample, it is viable with certain probability to evaluate range in which the value falls into.

This range is called the confidence interval of average and is given by the formula:

$$
M-t_{\alpha} \cdot \frac{S D}{\sqrt{n-1}}<m<M+t_{\alpha} \times \frac{S D}{\sqrt{n-1}}
$$

where:

$M$ - average

$t_{\alpha}$ - the value of the Student's t-distribution, depending on the assumed probability (usually 95\%) and sample size

$S D$ - Standard deviation

$n$ - number of sample

$m$ - actual average

According to Eq. (1) the accuracy of determining the actual average depends on the standard deviation of the sample, the number of measuring points and the value of the Student's t-distributions. The value of the Student's t-distributions is a function of the sample size.

Fig. 2. shows influence of number of samples on value of confidence interval relation to standard deviations of results. If two samples are taken, the confidence interval is more than 12 times greater than the standard deviation obtained. Confidence interval is equal to the standard deviation in case of 7 samples and further decrease with larger number of samples. 


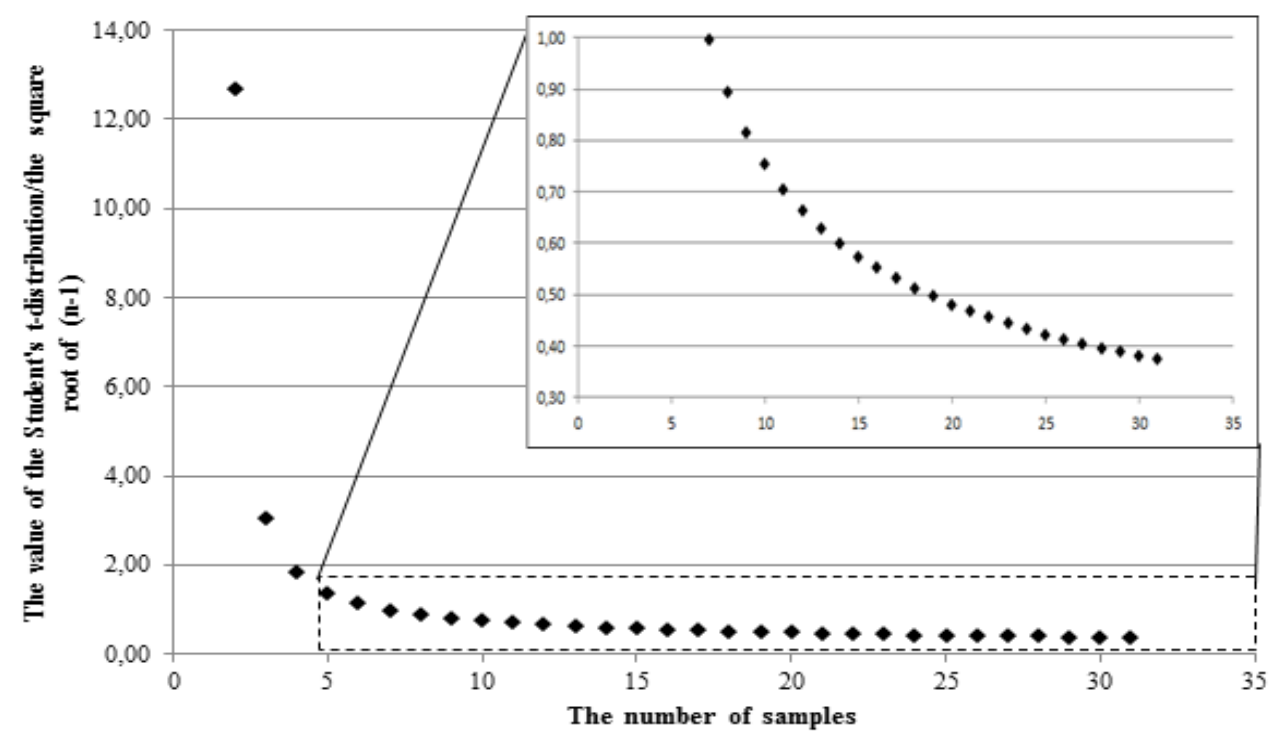

Fig. 2. Dependence of uncertainty in determining the actual average from the number of samples.

\section{Results}

\subsection{The first step of experiment - aeration}

Microscopic observations of protozoa lasted 250 minutes. Effect of aeration on the population of protozoa in the beakers was shown in Fig. 3. The amount of protozoa varies depending on presence or absence of aeration. It has been observed that in aerated beaker, number of protozoa was oscillating in range of $4000000-11000000 \mathrm{cells} / \mathrm{dm}^{3}$. On the contrary, the non-aerated beaker contained $4000000-7500000$ cells/ $\mathrm{dm}^{3}$ of protozoa.

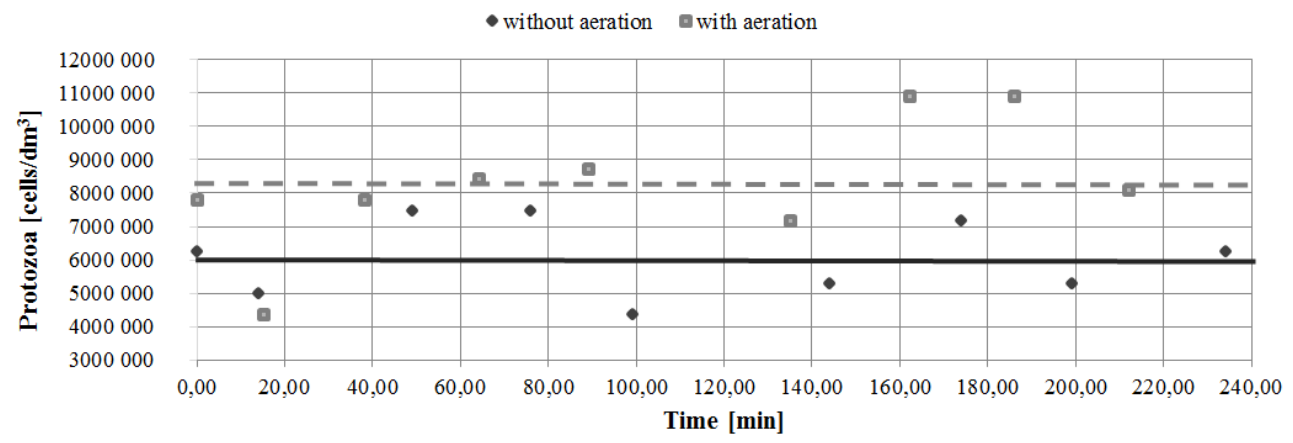

Fig. 3. Effect of aeration on the population protozoa.

Based on the results of the experiment, it can be observed, that the amount of protozoa in beaker with aeration was higher (the dashed line on the Fig. 3), than in beaker without aeration (the black line on the Fig. 3). The whole experiment lasted few hours. The statistical data are presented in Table 1. The average amount of microorganisms in the aerated probe was about 8264000 cells $/ \mathrm{dm}^{3}$, while in the non-aerated was 
6076000 cells $/ \mathrm{dm}^{3}$. Standard deviation in beaker with aeration was higher by 800000 in comparison to non-aerated probe. The difference might have been caused by protozoa dying due to non-aerated environment or protozoa accumulating inside sludge flocks.

The discrepancy in the results shows how different methods may give very different values. Without unified methodology, there is a risk of performing unreliable experiments. Therefore, clear and unified procedures should be precisely determined.

Table 1. Observation results in the sample aeration.

\begin{tabular}{|c|c|c|c|c|c|}
\hline \multirow[t]{2}{*}{ Beaker } & Average & $\begin{array}{l}\text { Standard } \\
\text { deviation }\end{array}$ & $\begin{array}{c}\text { The } \\
\text { number of } \\
\text { samples }\end{array}$ & $\begin{array}{l}\text { Confidence } \\
\text { interval for } \\
\text { the average }\end{array}$ & $\begin{array}{l}\text { The ratio of the } \\
\text { confidence interval } \\
\text { to the average }\end{array}$ \\
\hline & cells $/ \mathbf{d m}^{3}$ & cells $/ \mathbf{d m}^{3}$ & $\mathbf{n}$ & cells/dm ${ }^{3}$ & $\%$ \\
\hline Without aeration & 6076000 & 1084000 & 10 & 831000 & $14 \%$ \\
\hline With aeration & 8263000 & 1864000 & 10 & 1429000 & $17 \%$ \\
\hline
\end{tabular}

\subsection{The second stage of experiment - the number of samples}

The whole experiment lasted 12 days. Microscopic observations of microorganisms were carried out in selected 3 days. Each day 16 samples were taken. Fig. 4-6. show the results of observations as well as confidence interval values.

The average quantity of protozoa in the samples oscillates between $5000000-7300000$. The ratio of the confidence interval to mentioned average was in range of $7-30 \%$ depending of number of samples included in calculations.

Basing on the results of the experiment, it can be observed, that below 10 samples taken from beaker the ratio of the confidence interval to mentioned average was always higher than $10 \%$. The observation gave very accurate and reliable results for the number of samples from 10 to 13 . For this quantity of samples the ratio of the confidence interval to average was about $10 \%$ which is satisfactory.

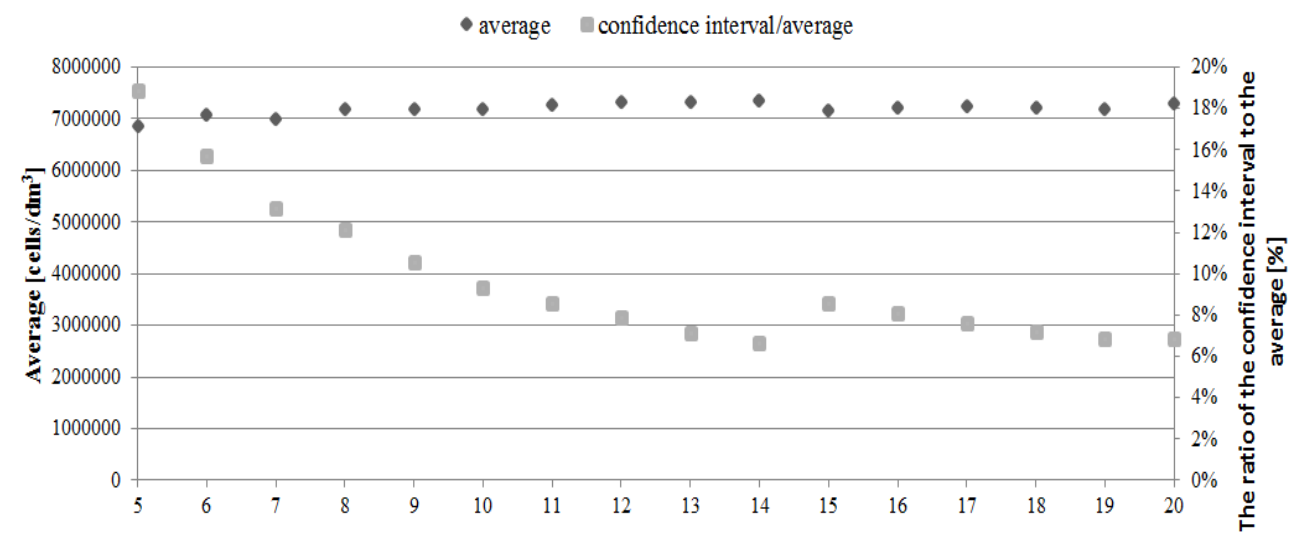

The number of samples

Fig. 4. Results of observation on $4^{\text {th }}$ day. 


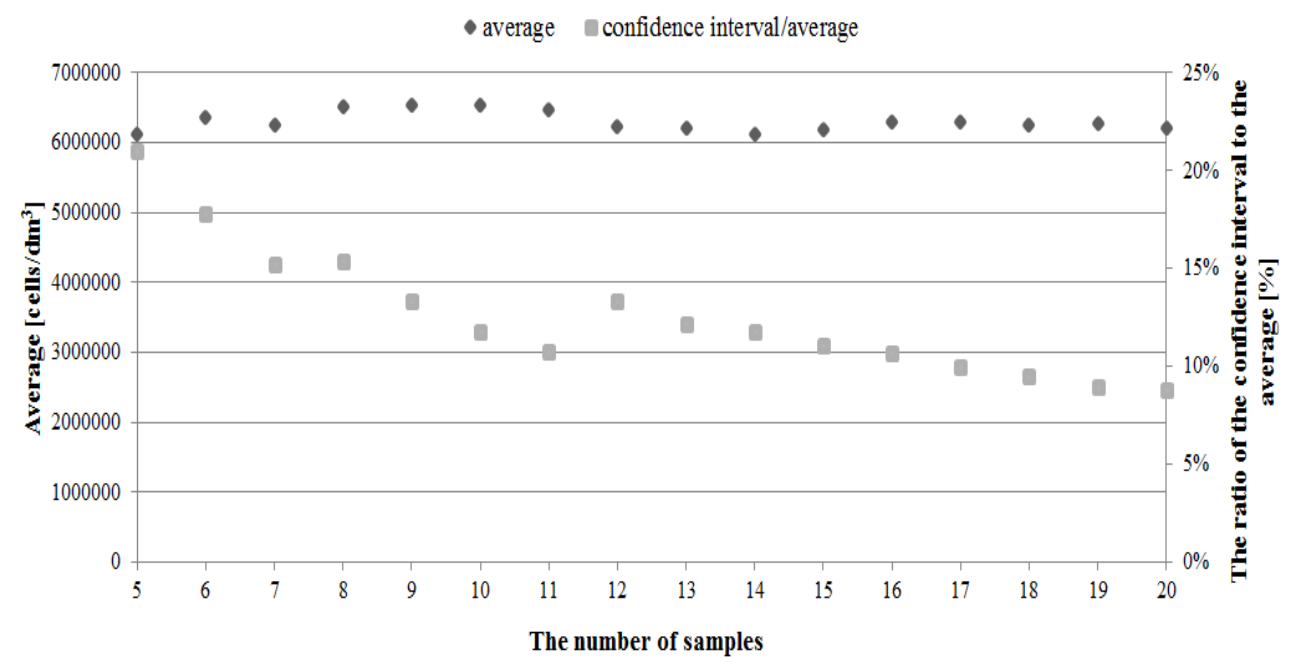

Fig. 5. Results of observation on $11^{\text {th }}$ day.

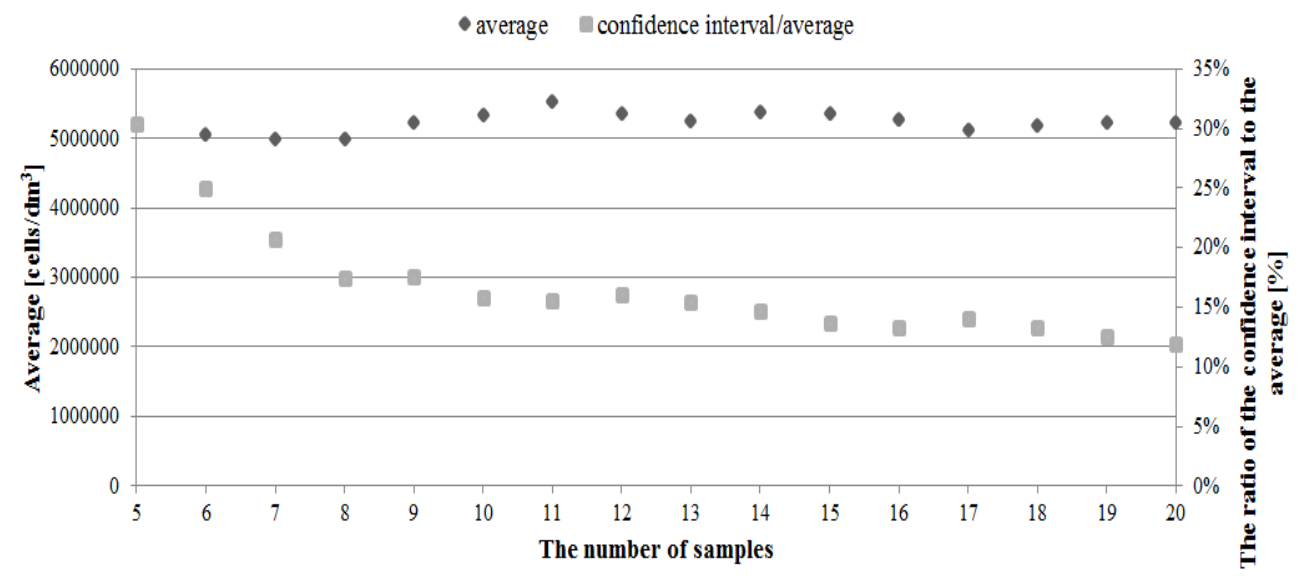

Fig. 6. Results of observation on $12^{\text {th }}$ day.

\section{Conclusions}

- The aeration of the beakers have positive influence on the amount of detected protozoa. The quantity of protozoa in beakers with aeration is higher than in beaker without aeration. Probably, the changes in amount of protozoa is caused by death of protozoa or their accumulation inside sludge flocks. The difference is 2200000 cells $/ \mathrm{dm}^{3}$ which is $25 \%$ of average of protozoa number in beaker with aeration.

- Differences in the results confirm thesis stated in this article. Method with aeration gives different data values in comparison to method without aeration. As a result, absence of unified procedures may cause critical errors in performed experiments. Therefore, it is important to standardize procedures of measuring protozoa quantity in sludge. 
- The second stage of experiment shows, that the lowest amount of samples which gives the acceptable results is from 10 to 13 . For those values, the ratio of the confidence interval to the average quantity of protozoa in the samples is about $10 \%$. The confidence interval with 10 and more samples is not changing significantly.

- Number of samples as well as calculations of confidence intervals should be standards and should be presented.

The authors gratefully acknowledge co-funding from the National Centre for Research and Development (grant no LIDER/16/0172/L-7/15/NCBR/2016).

\section{References}

1. P. Madoni, Italian Journal of Zoology 78, 1, 3-11 (2011)

2. Y. Lee, J.A. Oleszkiewicz, Water Research 37, 4202-4210 (2003)

3. M. Herrero, D.C. Stuckey Chemosphere 140, 119-128 (2015)

4. Y. Lifang, P. Dangcong, R. Yongxiang, Bioresource Technology 102, 10855-10860 (2011)

5. A.J. Pogue, K.A. Gilbride, Can. J. Microbiol. 53, 559-571 (2007) 